\title{
Structure and properties of a model conductive filament/host oxide interface in $\mathrm{HfO}_{2}$-based ReRAM
}

\author{
A. C. M. Padilha* and K. P. McKenna \\ Physics and Electronics Department, University of York, York, Heslington YO105DD, United Kingdom
}

(Received 15 February 2018; published 6 April 2018)

\begin{abstract}
Resistive random-access memory (ReRAM) is a promising class of nonvolatile memory capable of storing information via its resistance state. In the case of hafnium oxide-based devices, experimental evidence shows that a conductive oxygen-deficient filament is formed and broken inside of the device by oxygen migration, leading to switching of its resistance state. However, little is known about the nature of this conductive phase, its interface with the host oxide, or the associated interdiffusion of oxygen, presenting a challenge to understanding the switching mechanism and device properties. To address these problems, we present atomic-scale first-principles simulations of a prototypical conductive phase (HfO), the electronic properties of its interface with $\mathrm{HfO}_{2}$, as well as stability with respect to oxygen diffusion across the interface. We show that the conduction-band offset between $\mathrm{HfO}$ and $\mathrm{HfO}_{2}$ is $1.3 \mathrm{eV}$, smaller than typical electrode- $\mathrm{HfO}_{2}$ band offsets, suggesting that positive charging and band bending should occur at the conductive filament- $\mathrm{HfO}_{2}$ interface. We also show that transfer of oxygen across the interface, from $\mathrm{HfO}_{2}$ into $\mathrm{HfO}$, costs around $1.2 \mathrm{eV}$ per atom and leads to a gradual opening of the HfO band gap, and hence disruption of the electrical conductivity. These results provide invaluable insights into understanding the switching mechanism for $\mathrm{HfO}_{2}$-based ReRAM.
\end{abstract}

DOI: 10.1103/PhysRevMaterials.2.045001

\section{INTRODUCTION}

Metal-oxide-based resistive random-access memory (ReRAM) technologies promise fast read/write times, large on/off conductivity ratio, high endurance, as well as nonvolatility [1]. $\mathrm{HfO}_{2}$-based ReRAM in particular has been the focus of considerable research owing to its good chemical and thermal stability as well as compatibility with current silicon technology [1-6]. The resistance switching effect in $\mathrm{HfO}_{2}$ is thought to be due to the formation and rupture of an oxygen-deficient conductive filament $(\mathrm{CF})$ bridging between the electrodes [7-9]. Models suggest that on application of an electrical bias above a certain threshold, migration of oxygen ions into or out of the $\mathrm{CF}$ (from the surrounding host oxide or the electrode) reversibly oxidizes and reduces the filament, changing its resistivity [10-12] (Fig. 1). However, since the $\mathrm{CF}$ is buried inside the oxide matrix between electrodes, it is extremely challenging to probe experimentally, and many open questions remain concerning its structure as well as thermodynamic and electronic properties. In particular, very little is known about the interface between the CF and the host oxide $\left(\mathrm{HfO}_{2}\right)$. For example, the band offset between the $\mathrm{CF}$ and $\mathrm{HfO}_{2}$ is rarely discussed, but is important as any difference between the electrode- $\mathrm{HfO}_{2}$ and $\mathrm{CF}-\mathrm{HfO}_{2}$ band offsets will induce charge transfer and band bending within the filament, affecting electron transport and device parameters such as

\footnotetext{
*claudio.padilha@york.ac.uk

Published by the American Physical Society under the terms of the Creative Commons Attribution 4.0 International license. Further distribution of this work must maintain attribution to the author(s) and the published article's title, journal citation, and DOI.
}

the on/off ratio. The thermodynamics of oxygen transfer across the interface leading to changes in the electric transport properties of the CF is also not well understood. The absence of direct information on this key interface limits our ability to model, understand, and optimize ReRAM device operation.

In this paper, we present first-principles density functional theory (DFT) calculations to investigate the structure, electronic properties, and oxygen-transfer characteristics of an interface between a $\mathrm{CF}$ and the host oxide in $\mathrm{HfO}_{2}$. As noted above, there is little direct evidence on the structure of the CF in ReRAM devices since their nanoscale size and buried nature makes them very difficult to probe. However, theoretical calculations have identified a number of oxygen-deficient phases of $\mathrm{HfO}_{2}$ that are reasonably stable and conductive, including $\mathrm{HfO}, \mathrm{Hf}_{2} \mathrm{O}_{3}[13,14]$, as well as the limiting case of metallic Hf. In our calculations, we consider HfO as the model for the $\mathrm{CF}$ and build an atomistic model for a stable interface between $\mathrm{HfO}$ and $\mathrm{HfO}_{2}$. The calculated conduction-band offset (CBO) at the interface is $1.3 \mathrm{eV}$, considerably smaller than typical electrode- $\mathrm{HfO}_{2}$ band offsets (e.g., $3.4 \mathrm{eV}$ for $\mathrm{Pt}$ [15]). This suggests that for this kind of device, significant positive charging and band bending should occur in the CF near the $\mathrm{CF}-\mathrm{HfO}_{2}$ interface. Such band alignment is not extensively debated in the literature [16-18] even though it is key to understanding properties such as charge transport [19]. We also show that the energy cost to transfer oxygen from $\mathrm{HfO}_{2}$ lattice sites into interstitial sites in $\mathrm{HfO}$ increases almost linearly with the concentration of oxygen atoms $(1.2 \mathrm{eV}$ per transferred oxygen). Associated with the increasing concentration of interstitial oxygen is a gradual opening of the band gap in HfO, disrupting its originally conducting behavior. The energy cost for oxygen transfer from lattice sites in $\mathrm{HfO}_{2}$ is relatively high, suggesting that a reasonable amount of energy (e.g., thermal or electrical) should be provided for the system to switch via 


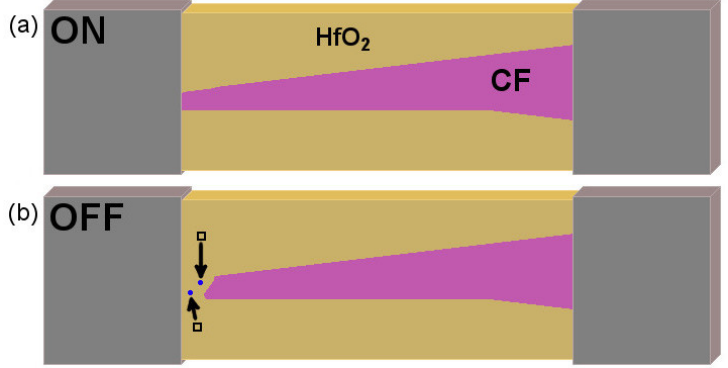

FIG. 1. Schematic of a $\mathrm{HfO}_{2}$-based ReRAM device. (a) In the ON state, an oxygen-deficient conductive filament (CF) connects the electrodes [dark (pink) region]. (b) Migration of oxygen atoms (small blue dots) from the host oxide $\left(\mathrm{HfO}_{2}\right)$ into the $\mathrm{CF}$ disrupts the conductive nature of the filament, resetting the device to the OFF state. The small black hollow squares represent the oxygen vacancies left behind in the $\mathrm{HfO}_{2}$ lattice. Subsequent out-diffusion of oxygen, filling the oxygen vacancies in the host oxide, restores the device to the ON state.

the oxygen-migration mechanism. However, the transfer of oxygen from interstitial sites in $\mathrm{HfO}_{2}$ (which may be populated during forming under high bias) or from the electrode would be much more facile. Altogether, these results provide invaluable insights into a prospective switching mechanism for $\mathrm{HfO}_{2}$ based ReRAM.

\section{METHODS}

The electronic structure calculations as well as structural relaxations performed for this work are based on DFT and were carried out using the VASP code [20-22]. The $5 p 6 s 5 d$ electrons of $\mathrm{Hf}$ and the $2 s 2 p$ electrons of $\mathrm{O}$ were considered as valence electrons and their interaction with the core electrons were described using the projector augmented waves (PAW) method [23]. We used exchange-correlation functionals proposed by Perdew, Burke, and Ernzerhof (PBE) [24] for all relaxations (forces on atoms $<2.5 \times 10^{-2} \mathrm{eV}^{-1}$ ), while the hybrid functional proposed by Heyd, Scuseria, and Ernzerhof (HSE) [25] was used to obtain the electronic structure for the bulk materials after relaxation. The band structures of $\mathrm{HfO}_{2}$ and $\mathrm{HfO}$ were obtained using the HSE functional for the bulk pristine materials, while the electronic bands for the supercell calculations containing interstitial oxygen in HfO were obtained using the PBE functional and unfolded by use of the BANDUP program [26,27]. The cutoff energies and $k$-point sampling were set, respectively, to $350 \mathrm{eV}$ and $\Gamma$-only for the supercell calculations (see Sec. III A), $450 \mathrm{eV}$ and $6 \times 6 \times 4 \Gamma$-centered for bulk $\mathrm{HfO}_{2}$, and $450 \mathrm{eV}$ and $6 \times 6 \times 8 \Gamma$-centered for bulk HfO calculations. This choice of parameters was such that we ensured total energy convergence of the order of $10 \mathrm{meV}$ for all systems.

To model the interface between $\mathrm{HfO}-\mathrm{HfO}_{2}$, we used a supercell approach (details of the construction of the supercell are given in Sec. III A). The following procedure was employed to determine the valence-band offset (VBO) between $\mathrm{HfO}_{2}$ and HfO: (i) The structure of the interface supercell was first optimized using the PBE functional. (ii) The Hartree potential averaged within planes perpendicular to the interface was computed and compared to corresponding plane-averaged potentials for the bulk $\mathrm{HfO}_{2}$ and $\mathrm{HfO}$ unit cells to identify bulklike regions in the supercell. (iii) By computing average values of the Hartree potential in the bulklike regions, we were able to align the calculated band structures for bulk $\mathrm{HfO}_{2}$ and $\mathrm{HfO}$ to a common reference in the supercell. Examples of the application of this procedure to other materials can be found in the literature $[16,28]$.

\section{RESULTS}

\section{A. Construction of the interface}

In order to model the interface between the filament and the $\mathrm{HfO}_{2}$ surrounding matrix, we initially considered a number of oxygen-deficient phases, such as $\mathrm{Hf}_{2} \mathrm{O}_{3}, \mathrm{HfO}, \mathrm{Hf}_{2} \mathrm{O}$, and $\mathrm{Hf}_{3} \mathrm{O}[13,14]$, for the $\mathrm{CF}$ and the three polymorphs of $\mathrm{HfO}_{2}$ : low-temperature monoclinic $\left(\mathrm{m}-\mathrm{HfO}_{2}\right)$ [29], intermediatetemperature tetragonal $\left(\mathrm{t}-\mathrm{HfO}_{2}\right)$, and high-temperature cubic (c- $\mathrm{HfO}_{2}$ ) for the host oxide. Of these possible combinations, the c-HfO- $\mathrm{HfO}_{2}$ interface is the most suited for theoretical modeling owing to symmetry compatibility (the Hf sublattice of both structures has a hexagonal symmetry), small lattice mismatch ( $<2.8 \%$ strain), and computationally feasible number of atoms in the supercell (452). Although c- $\mathrm{HfO}_{2}$ is not the most stable phase at ambient conditions, both $\mathrm{c}-\mathrm{HfO}_{2}$ and $\mathrm{t}-$ $\mathrm{HfO}_{2}$ are reported to be present in $\mathrm{HfO}_{2}$ thin films [30] and have a similar electronic structure to the low-temperature phase. For these reasons, $\mathrm{c}-\mathrm{HfO}_{2}$ is expected to be a reasonable model for the host oxide. HfO was first identified as a conductive and reasonable stable oxygen-deficient phase by Zhang et al. using evolutionary algorithms [13]. They characterized it as a metastable Hf-O compound at ambient pressure, lying only slightly above the convex hull. The electronic structure was also studied, using both PBE and HSE functionals, and they concluded that it was a semiconductor with a very small band gap of $E_{g} \approx 0.1 \mathrm{eV}$. Our HSE calculations (see Sec. IIIB) resulted in $E_{g}=0.07 \mathrm{eV}$. Therefore, since HfO is a much better conductor than $\mathrm{HfO}_{2}$, it represents a reasonable model for the $\mathrm{CF}$.

The transformation of $\mathrm{c}-\mathrm{HfO}_{2}$ from its original cubic unit cell into the hexagonal cell was performed in a sequence of steps. We started by positioning the [111] direction of the original cubic cell along the $c$ direction of the new hexagonal cell, and used the Hf-Hf distances as the $a$ and $b$ vectors. This was possible due to the fact that the $\mathrm{Hf}$ and $\mathrm{O}$ sublattices in the $\mathrm{c}-\mathrm{HfO}_{2}$ cell are made of hexagonal sublattices perpendicular to the [111] direction. The resulting cell, which will be referred to as $\mathrm{HfO}_{2}$ from now on, is shown in Fig. 2(a) and the calculated lattice parameters are presented in Table I. Having selected the two materials, the next step was to create the supercell containing the $\mathrm{HfO}-\mathrm{HfO}_{2}$ interface. First, we created a $3 \times 3 \times$ 4 supercell of $\mathrm{HfO}_{2}$ and combined it with a $2 \times 2 \times 6$ supercell of HfO by matching their $a$ and $b$ vectors. The structures were repeated along the $c$ direction in order to observe a bulklike region in the center of each layer. Finally, the spacing and orientation between the two layers was carefully selected such that the bonding pattern does not deviate considerably from the bulk materials. This helps to avoid void regions or atoms that are too close to each other. The resulting supercell containing 


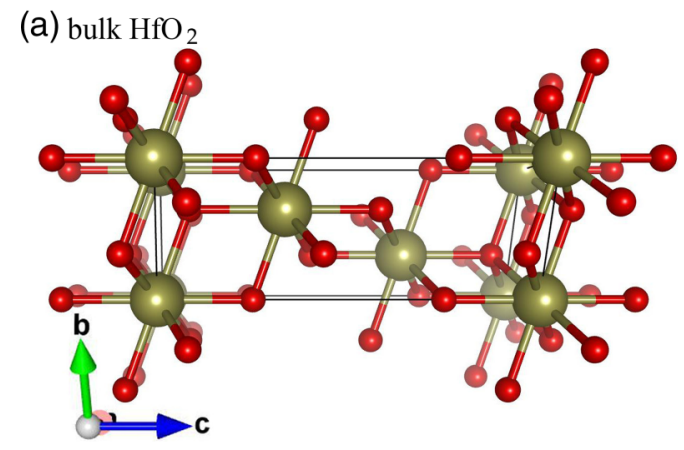

(b) bulk HfO

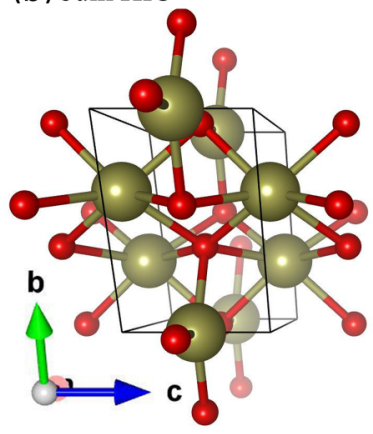

(c)

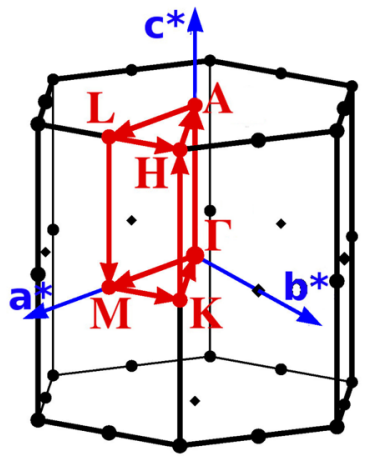

$\mathrm{HfO}_{2}$

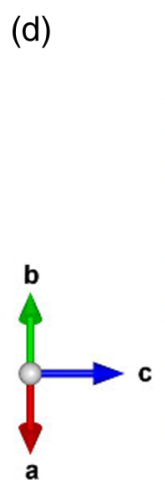

$\mathrm{HfO}_{2}$ HfO

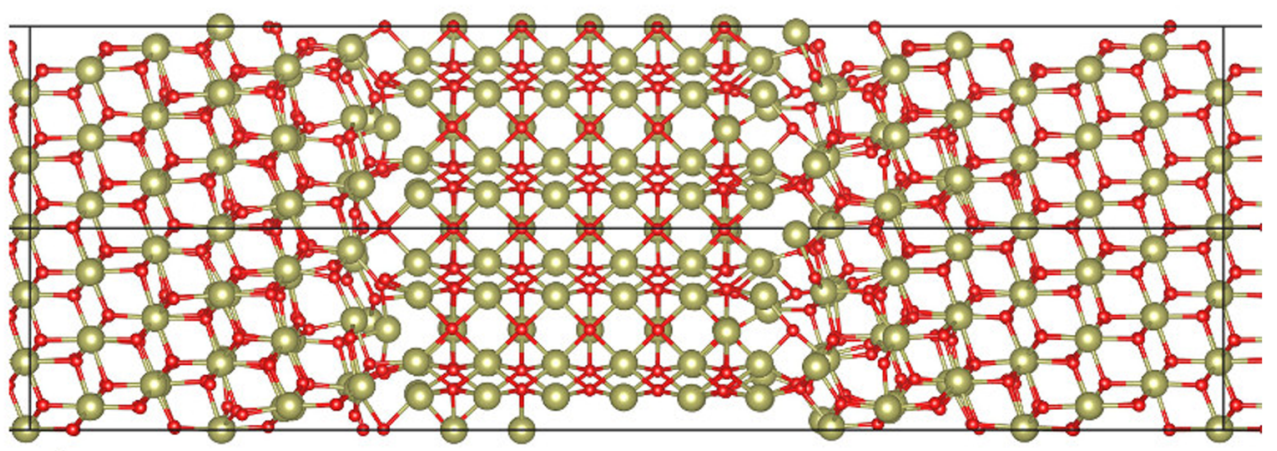

(e)

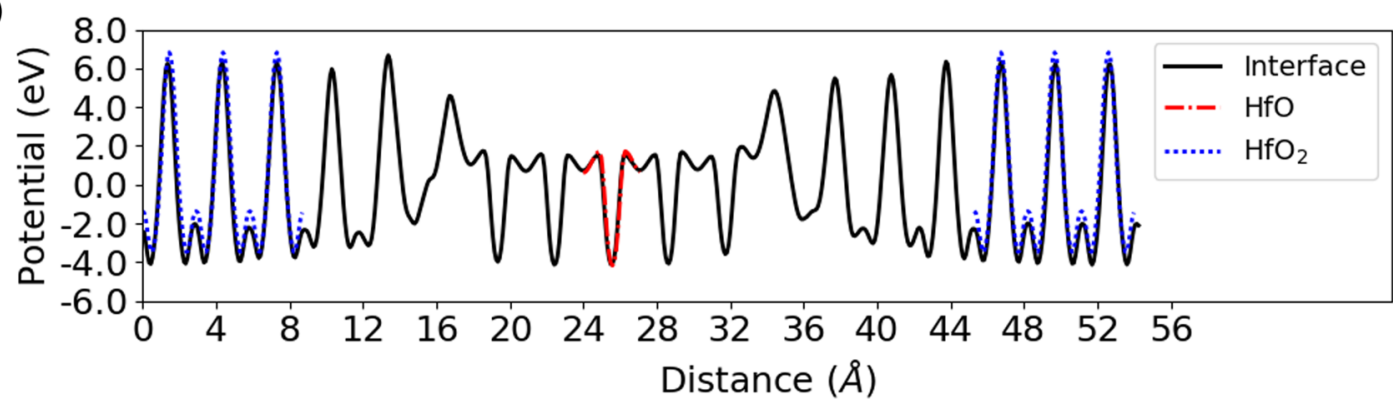

FIG. 2. (a) Hexagonal bulk $\mathrm{HfO}_{2}$ unit cell obtained from the cubic structure. (b) Hexagonal bulk HfO unit cell. (c) Brillouin zone of the respective hexagonal cells, where the path for the band structure calculations is highlighted and the points of high symmetry are labeled. (d) Supercell containing the interface between $\mathrm{HfO}_{2}$ and $\mathrm{HfO}$. Large golden spheres are $\mathrm{Hf}$ atoms, while small red spheres are $\mathrm{O}$ atoms. (e) Plane-averaged Hartree potential along the $c$ direction of the supercell containing the interface (solid black line), the $\mathrm{HfO}_{2}$ cell (dotted blue line), and the HfO cell (dot-dashed red line).

the interface, after relaxation, resulted in the lowest adhesion energy of all the other candidates, $E_{\mathrm{a}}=-0.11 \mathrm{eV} \AA^{-2}$, and is shown in Fig. 2(d).

TABLE I. Structural parameters for both $\mathrm{HfO}$ and $\mathrm{c}-\mathrm{HfO}_{2}$ after the transformation from cubic to hexagonal unit cells. Values correspond to the relaxed structures using both PBE and HSE functionals.

\begin{tabular}{lccccc}
\hline \hline & & $a(\AA)$ & $c(\AA)$ & $\alpha\left({ }^{\circ}\right)$ & $\gamma\left({ }^{\circ}\right)$ \\
\hline HfO & PBE & 5.225 & 3.184 & 90.00 & 120.00 \\
& HSE & 5.180 & 3.152 & 90.00 & 120.00 \\
$\mathrm{HfO}_{2}$ & PBE & 3.582 & 8.774 & 90.00 & 120.00 \\
& HSE & 3.550 & 8.700 & 90.00 & 120.00 \\
\hline \hline
\end{tabular}

\section{B. Electronic structure of $\mathrm{HfO}$ and its band alignment with $\mathrm{HfO}_{2}$}

The band structure of $\mathrm{HfO}$ obtained with both the PBE and HSE functionals is presented in Figs. 3(a) and 3(b). It can be seen that the HSE calculation results in a nearly zero band gap $\left(E_{g}=0.07 \mathrm{eV}\right)$, while PBE describes the system as a metal, in accordance with the results obtained by Zhang et al. The effect of the hybrid functional was a slight rigid shift of the higherenergy, unoccupied electronic bands, while the dispersion of the bands around the Fermi energy was nearly identical to the bands obtained by the PBE functional. The underestimation of the band gaps of semiconductors and insulators is a well-known issue with semilocal exchange-correlation functionals (such as PBE) [31].

Owing to the importance of the band alignment for the ReRAM application, we used DFT calculations to elucidate the 
(a) $\mathrm{HfO}$ - PBE

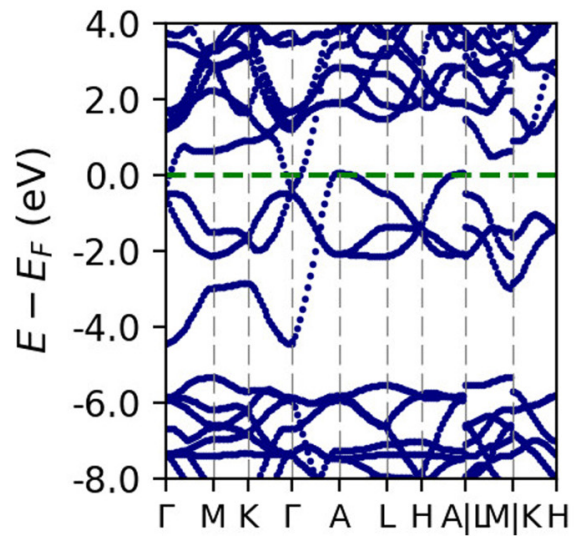

(b) HfO - HSE

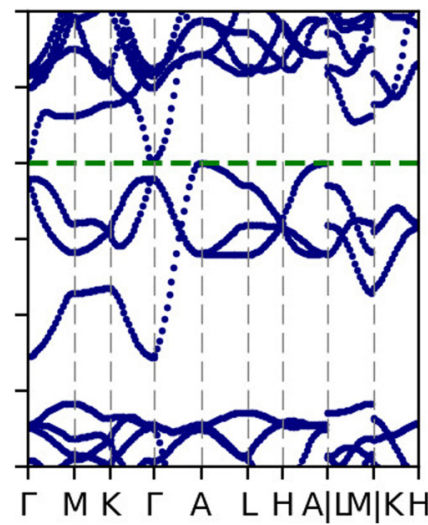

(c) $\mathrm{HfO}_{2}-\mathrm{HSE}$

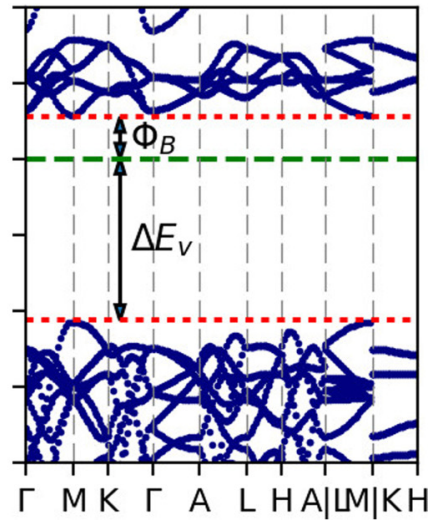

FIG. 3. Band structure of HfO calculated using (a) $\mathrm{PBE}$ and (b) $\mathrm{HSE}$ functionals. (c) $\mathrm{HfO}_{2}$ band structure calculated using the HSE functional. Both HSE calculations were aligned using $\Delta E_{v}=4.26 \mathrm{eV}$. The Schottky barrier $\left(\Phi_{B}=1.3 \mathrm{eV}\right)$ is also shown. The Fermi energy $\left(E_{F}\right)$ of $\mathrm{HfO}$ is set to zero.

properties of this system, using the method described in Sec. II. Using this approach, we obtained a VBO of $\Delta E_{v}=4.26 \mathrm{eV}$. The aligned band structure of $\mathrm{HfO}$ and $\mathrm{HfO}_{2}$ calculated using the HSE functional is shown in Figs. 3(b) and 3(c). From the aligned band structures, we can see that there is a Schottky barrier of $\Phi_{B}=1.3 \mathrm{eV}$ at the $\mathrm{HfO}-\mathrm{HfO}_{2}$ interface. This result is similar to that found in previous studies of the $\mathrm{Hf}_{2} \mathrm{O}_{3}-\mathrm{HfO}_{2}$ and $\mathrm{Hf}-\mathrm{HfO}_{2}$ interfaces: 1.96 and $0.61 \mathrm{eV}$, respectively [17]. Typical conduction-band offsets for the $\mathrm{HfO}_{2}$-electrode interfaces are much higher (e.g., Kumar et al. found $\Phi_{B}=3.4 \mathrm{eV}$ for a $\mathrm{Pt}-\mathrm{HfO}_{2}$ interface [15]). Therefore, one can expect significant band bending and corresponding positive-charge buildup to take place at the $\mathrm{HfO}-\mathrm{HfO}_{2}$ interface, leading to a strong rectifying behavior, in line with the large on/off ratios reported in the literature [1].

\section{Oxygen interdiffusion}

The migration of oxygen ions from and into the $\mathrm{CF}$ is believed to be the main mechanism of resistance switching in $\mathrm{HfO}_{2}$-based ReRAM. The radial movement of such oxygen ions around the $\mathrm{CF}$ in a $\mathrm{HfO}_{2}$-based ReRAM device during switching operation was recently detected by Kumar et al. [12]. Aiming to understand if such process would explain the functioning of the device, we assessed its stability by means of total-energy calculations for the interface supercell discussed above. We started by randomly selecting pairs composed of one oxygen atom from the $\mathrm{HfO}_{2}$ layer and one interstitial position from the HfO layer (identified by using the cages tool of the AFLOW online system [32]). Next, we placed the oxygen into the interstitial position, creating an extended Frenkelpair defect across the interface, in the same way as reported previously for $\mathrm{HfO}_{2}$-metal interfaces $[33,34]$. For each number of pairs created, from a single pair up to seven pairs, we relaxed and calculated the total energies for 32 structures and obtained their formation energy $(\Delta E)$ with respect to the pristine supercell.

The average formation energy as a function of the number of extended Frenkel pairs is shown in Fig. 4. The linear trend suggests that the energy required to create additional pairs is constant at around $1.2 \mathrm{eV}$ per defect pair, at least for a small number of such defects. Similar formation energies for extended Frenkel pairs have been obtained for $\mathrm{HfO}_{2} /$ electrode interfaces in previous DFT studies: $\mathrm{t}-\mathrm{HfO}_{2}-\mathrm{Mo}(1.5 \mathrm{eV})$ [34], $\mathrm{m}-\mathrm{HfO}_{2}-\mathrm{Rh}(2.8-4.1 \mathrm{eV})$, and c- $\mathrm{HfO}_{2}-\mathrm{Ni}(1.2-3.1 \mathrm{eV})$ [33]. Traore et al. also considered multiple extended Frenkel pairs at the $\mathrm{m}-\mathrm{HfO}_{2}-\mathrm{Ti}$ interface with formation energies raging from -0.08 to $1.04 \mathrm{eV}$ for one to 10 pairs [35].

After understanding the energetic stability with respect to the migration of the oxygen ions into the HfO structure, we turned our attention to the impact of such migration on the electronic structure of HfO. We built $2 \times 2 \times 2$ supercells made of repetitions of both the pristine unit cell and the unit cell with the interstitial. For example, for a single O interstitial, we used one unit cell containing a single interstitial atom and the remaining seven cells were the pristine one. For higher concentrations of the defect, we simply replaced additional

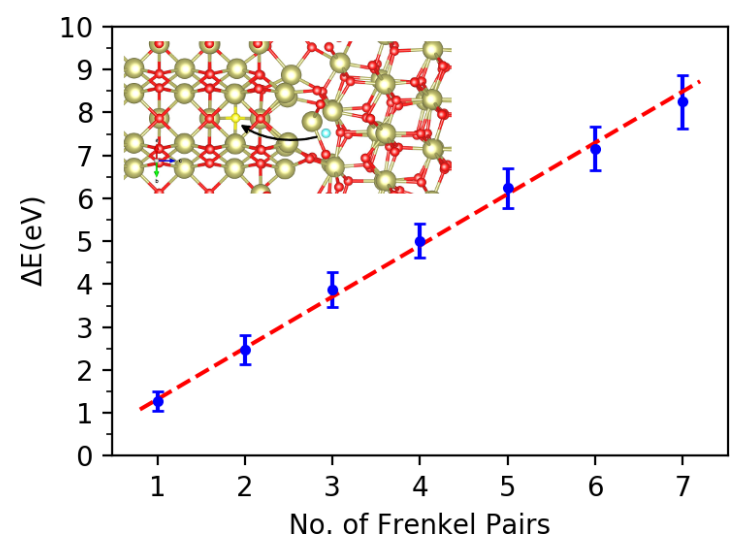

FIG. 4. Formation energy $(\Delta E)$ as a function of the number of extended Frenkel pairs across the $\mathrm{HfO}-\mathrm{HfO}_{2}$ interface. Each data point is an average over 32 structural configurations and the error bars indicate their standard deviations. The dashed red line is a linear fit. The inset shows an example of an extended Frenkel pair, where the light blue sphere on the right $\left(\mathrm{HfO}_{2}\right)$ represents an oxygen vacancy created by the displacement of an oxygen ion into the interstitial position in $\mathrm{HfO}$ (yellow sphere). 

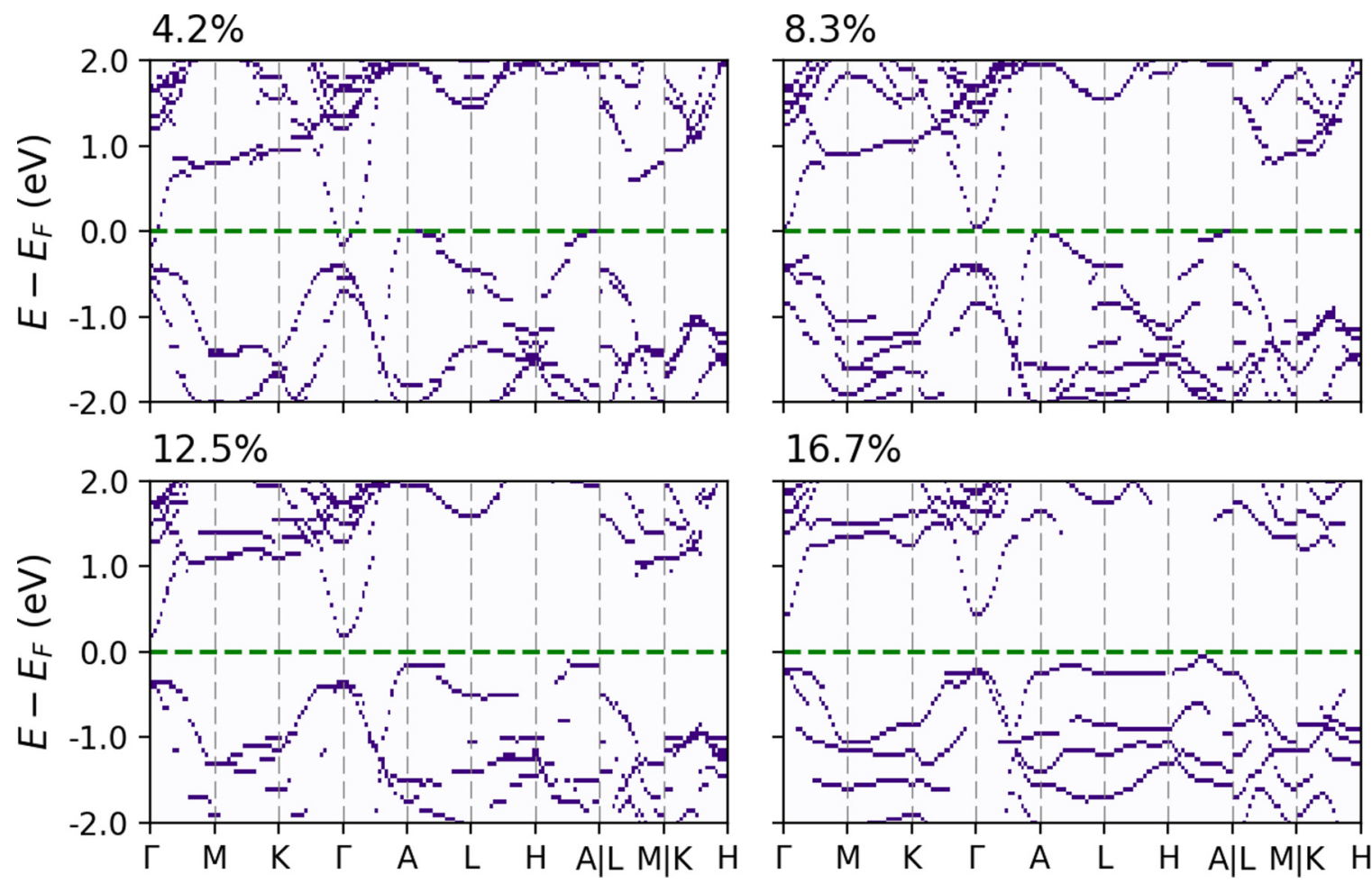

FIG. 5. Unfolded band structure of HfO obtained using the PBE functional for various concentrations of interstitial O atoms. The Fermi energy $\left(E_{F}\right)$ is used as reference.

pristine cells by the cells containing a single interstitial in order to model concentrations of $4.2 \%, 8.3 \%, 12.5 \%$, and $16.7 \%$ interstitial oxygen content for $1,2,3$, and 4 interstitials, respectively.

One drawback in this approach is that since the unit-cell vectors are expanded by a factor of two along each dimension, the corresponding reciprocal cell vectors are reduced by the same factor, resulting in the phenomena known as band folding of the Brillouin zone [36]. In order to overcome this issue, we used the band-unfolding method as implemented in the BANDUP program [26,27]. The resulting band structures are presented in Fig. 5. As the oxygen concentration increases, the $\Gamma-A$ band gap widens due to the shift to higher energies of the unoccupied electronic band at the $\Gamma$ point. At the limiting situation, where all interstitial sites are occupied, our HSE calculation predicts a band gap of $1.1 \mathrm{eV}$. This shows that the migration of oxygen into the oxygen-deficient HfO phase is responsible for a change in its conducting properties, providing a model for the resistive switching effect observed in ReRAM devices.

\section{DISCUSSION}

Our results for the band alignment show good agreement with other theoretical studies. The interface between $\mathrm{HfO}_{2}$ and the $\mathrm{CF}$ was recently modeled by $\mathrm{Wu}$ et $\mathrm{al}$., who chose $\mathrm{Hf}_{2} \mathrm{O}_{3}$ and metallic $\mathrm{Hf}$ as the materials for the filament and cubic $\mathrm{HfO}_{2}$ for the surrounding media. They found Schottky barriers in both interfaces to be $\Phi_{B}=1.96$ and $0.61 \mathrm{eV}$, respectively [17], while our result, $\Phi_{B}=1.3 \mathrm{eV}$, lies between both values. This points to the fact that the interface between the $\mathrm{CF}$ and the $\mathrm{HfO}_{2}$ matrix is indeed a Schottky contact.
Comparison with a typical electrode-oxide interface, such as $\mathrm{HfO}_{2} / \mathrm{Pt}$, which presents $\Phi_{B}=3.3 \mathrm{eV}$ [15], leads to the conclusion that a strong band bending would take place at the $\mathrm{HfO}-\mathrm{HfO}_{2}$ interface. As a consequence, a positive charge buildup would form within a depletion region, which encloses the $\mathrm{CF}$ and plays two main roles: (i) creation of a barrier which is responsible for keeping the charge carriers confined to the filament region, thus restricting the current to that small region, and (ii) appearance of a strong rectifying barrier at the tip of the filament, presenting resistance for the electronic transport into or from the electrode direction. This behavior could, in principle, be controlled by changing the electrode potential (i.e., its Fermi energy), altering the band-bending profiles at the $\mathrm{HfO}-\mathrm{HfO}_{2}$ interface, and consequently affecting key processes such as the oxygen interdiffusion. Experimental evidence is still required in order to confirm these predictions.

One important issue regarding the band alignment calculation shown here is the impact of the strain on the electronic properties of the system under study. For instance, a compressive strain could decrease the average bond lengths, leading to an increased electronic density which could result in higher energies of the electrostatic potential and/or higher eigenvalues for the electronic states of the system. To take this issue into consideration, we used the method proposed by Li et al. to calculate the deformation potentials [37] and estimated the changes in the valence band as well as the average electrostatic potential in all cases to be smaller than $0.1 \mathrm{eV}$. Therefore, we can safely neglect such corrections in our calculations since they would not change our interpretation of the results.

Experimental characterization of the $\mathrm{CF}$ material would be invaluable, but little is currently known. Celano et al., 
using atomic-force-microscopy-based tomography [38], and Li et al., using electron holography and transmission electron microscopy [39], have probed the CF. However, they only describe it as oxygen deficient and no characterization of the structure is presented. The choice of $\mathrm{HfO}$ as a model for the filament was considered good since we could show that this material presents a decrease in its electronic conductivity due to insertion of oxygen into its structure, owing to an increase of the band gap from virtually zero to $\approx 1 \mathrm{eV}$. From a semiconductor perspective, this increase would lead to a huge decrease of carriers and, in turn, to a change of orders of magnitude in the conductivity of the material, which is in line with many experimental results [1]. A phase transition (i.e., from $\mathrm{HfO}$ to $\mathrm{HfO}_{2}$ ) could also be another explanation for the switching, but is beyond the scope of this work.

One of the major drawbacks of the model presented in this work is the lack of a kinetic description of the process. We have reported total-energy calculations for the stability of the HfO structure upon insertion of the oxygen atoms, but the minimum-energy paths (MEPs) for their migration across the interface could not be determined due to the many possible pathways and the corresponding computational cost of such large investigation. A future work would be the screening of the possible paths by means of a metric such as formation energies of the isolated defects and/or distances between these defects, and obtaining the MEPs using nudged elastic-band (NEB) calculations. These results would provide the barrier heights for the migration of oxygen atomic species, which would be used as inputs for a kinetic Monte Carlo model capable of describing the evolution of the system.

\section{CONCLUSIONS}

We have studied the $\mathrm{HfO}_{2}$-CF interface of $\mathrm{HfO}_{2}$-based ReRAM devices using density functional theory calculations. We identified HfO as a prototypical model for the material composing the $\mathrm{CF}$ inside the active layer of the device since it presents a virtually zero band gap that can be made larger (up to $1.1 \mathrm{eV}$ ) upon insertion of oxygen atoms into interstitial positions. We then built a supercell containing the interface between $\mathrm{HfO}$ and $\mathrm{HfO}_{2}$, providing a model of the interface between the conductive filament and the surrounding oxide layer.
Calculation of the band alignment predicts that a Schottky barrier of $\Phi_{B}=1.3 \mathrm{eV}$ is present at the $\mathrm{HfO}-\mathrm{HfO}_{2}$ interface. This result is of particular importance since a large on/off resistance ratio $\left(>10^{5}\right)$ was observed for $\mathrm{HfO}_{2}$-based devices, which is expected of this kind of rectifying contact, which was also suggested by other theoretical studies. By comparison with typical oxide-electrode contacts, such as $\mathrm{HfO}_{2} / \mathrm{Pt}$, which present a larger barrier $\left(\Phi_{B}=3.4 \mathrm{eV}\right)$, we identify a large band bending at the oxide-CF interface, which would confine carriers inside the filament as well as present a rectifying barrier for their movement at the tip of the CF.

The interdiffusion of oxygen across the interface was assessed from a total-energy point of view, revealing itself as a feasible process since the insertion of oxygen atoms into the $\mathrm{CF}$ presents an approximately constant-energy cost $(1.2 \mathrm{eV}$ per inserted oxygen). This process in turn leads to the appearance of a small band gap in the HfO, which decreases its conductivity quite drastically and could consequently lead to resistance switching. We note that extended defect Frenkel formation across the $\mathrm{HfO}-\mathrm{HfO}_{2}$ interface is much more favorable than the equivalent process in $\mathrm{HfO}_{2}$ [40].

Overall, we have provided some insights on the resistance switching process, which, according to our results, could be explained by the oxygen migration across the HfO- $\mathrm{HfO}_{2}$ interface. Further studies, however, are still necessary in order to provide a comprehensive model capable of explaining the working principles of the devices, either by exploring the kinetics of the ionic movement or by experimental characterization of the conductive filaments.

All data created during this research are available by request from the University of York Research database [41].

\section{ACKNOWLEDGMENTS}

K.P.M. acknowledges support from EPSRC (Grant No. EP/K003151/1). This work made use of the facilities of Archer, the UK's national high-performance computing service, via our membership in the UK HPC Materials Chemistry Consortium, which is funded by EPSRC (Grant No. EP/L000202/1). This work also made use of the facilities of the N8 HPC Centre of Excellence, provided and funded by the $\mathrm{N} 8$ consortium and EPSRC (Grant No. EP/K000225/1). The Centre is coordinated by the Universities of Leeds and Manchester.
[1] F. Pan, S. Gao, C. Chen, C. Song, and F. Zeng, Mater. Sci. Eng., R 83, 1 (2014).

[2] J. H. Choi, Y. Mao, and J. P. Chang, Mater. Sci. Eng., R 72, 97 (2011).

[3] F. Nardi, S. Balatti, S. Larentis, D. C. Gilmer, and D. Ielmini, IEEE Trans. Electron Devices 60, 70 (2013).

[4] H.-S. P. Wong, H.-Y. Lee, S. Yu, Y.-S. Chen, Y. Wu, P.-S. Chen, B. Lee, F. T. Chen, and M.-J. Tsai, Proc. IEEE 100, 1951 (2012)

[5] J. Lee, J. Shin, D. Lee, W. Lee, S. Jung, M. Jo, J. Park, K. P. Biju, S. Kim, S. Park, and H. Hwang, Technical Digest - International Electron Devices Meeting, IEDM (IEEE, San Francisco, CA, USA, 2010), pp. 452-455.
[6] E. Miranda, C. Walczyk, C. Wenger, and T. Schroeder, IEEE Electron Device Lett. 31, 609 (2010).

[7] D.-H. Kwon, K. M. Kim, J. H. Jang, J. M. Jeon, M. H. Lee, G. H. Kim, X.-S. Li, G.-S. Park, B. Lee, S. Han, M. Kim, and C. S. Hwang, Nat. Nanotech. 5, 148 (2010).

[8] J. P. Strachan, M. D. Pickett, J. J. Yang, S. Aloni, A. L. David Kilcoyne, G. Medeiros-Ribeiro, and R. Stanley Williams, Adv. Mater. 22, 3573 (2010).

[9] G. Hwan Kim, J. Ho Lee, J. Yeong Seok, S. Ji Song, J. Ho Yoon, K. Jean Yoon, M. Hwan Lee, K. Min Kim, H. Dong Lee, S. Wook Ryu, T. Joo Park, and C. Seong Hwang, Appl. Phys. Lett. 98, 262901 (2011).

[10] R. Waser and M. Aono, Nat. Mater. 6, 833 (2007). 
[11] E. Hildebrandt, J. Kurian, and L. Alff, J. Appl. Phys. 112, 114112 (2012).

[12] S. Kumar, Z. Wang, X. Huang, N. Kumari, N. Davila, J. P. Strachan, D. Vine, A. L. D. Kilcoyne, Y. Nishi, and R. S. Williams, Appl. Phys. Lett. 110, 103503 (2017).

[13] J. Zhang, A. R. Oganov, X. Li, K. H. Xue, Z. Wang, and H. Dong, Phys. Rev. B 92, 184104 (2015).

[14] K. H. Xue, P. Blaise, L. R. C. Fonseca, and Y. Nishi, Phys. Rev. Lett. 110, 065502 (2013).

[15] A. Kumar, S. Mondal, and K. S. R. K. Rao, Appl. Phys. A 122, 1027 (2016).

[16] A. C. M. Padilha, A. R. Rocha, and G. M. Dalpian, Phys. Rev. Appl. 3, 024009 (2015).

[17] Z.-H. Wu, K.-H. Xue, and X.-S. Miao, J. Comput. Electron. 16, 1057 (2017).

[18] H. Li, Z. Zhang, and L. P. Shi, J. Electron. Mater. 45, 1142 (2016).

[19] S. B. Zhang, S.-H. Wei, and A. Zunger, Phys. Rev. Lett. 84, 1232 (2000).

[20] G. Kresse and J. Hafner, Phys. Rev. B 47, 558 (1993).

[21] G. Kresse and J. Hafner, Phys. Rev. B 49, 14251 (1994).

[22] G. Kresse and J. Furthmüller, Phys. Rev. B 54, 11169 (1996).

[23] P. E. Blöchl, Phys. Rev. B 50, 17953 (1994).

[24] J. P. Perdew, K. Burke, and M. Ernzerhof, Phys. Rev. Lett. 77, 3865 (1996).

[25] J. Heyd, G. E. Scuseria, and M. Ernzerhof, J. Chem. Phys. 118, 8207 (2003).

[26] P. V. C. Medeiros, S. Stafström, and J. Björk, Phys. Rev. B 89, 041407 (2014).

[27] P. V. C. Medeiros, S. S. Tsirkin, S. Stafström, and J. Björk, Phys. Rev. B 91, 041116 (2015).
[28] Y. H. Li, A. Walsh, S. Chen, W. J. Yin, J. H. Yang, J. Li, J. L. F. Da Silva, X. G. Gong, and S. H. Wei, Appl. Phys. Lett. 94, 212109 (2009).

[29] R. Ruh and P. W. R. Corfield, J. Am. Ceram. Soc. 53, 126 (1970).

[30] H.-Y. Lee, P.-S. Chen, C.-C. Wang, S. Maikap, P.-J. Tzeng, C.-H. Lin, L.-S. Lee, and M.-J. Tsai, Jpn. J. Appl. Phys. 46, 2175 (2007).

[31] J. P. Perdew and K. Schmidt, AIP Conf. Proc. 577120 (2001).

[32] R. V. Chepulskii and S. Curtarolo, Phys. Rev. B 79, 134203 (2009).

[33] O. Sharia, K. Tse, J. Robertson, and Alexander A. Demkov, Phys. Rev. B 79, 125305 (2009).

[34] A. A. Demkov, Phys. Rev. B 74, 085310 (2006).

[35] B. Traore, P. Blaise, B. Sklenard, E. Vianello, B. Magyari-Kope, and Y. Nishi, IEEE Trans. Electron Devices 65, 507 (2018).

[36] N. W. Ashcroft and N. D. Mermin, Solid State Physics (Saunders College, Philadelphia 1976).

[37] Y. H. Li, X. G. Gong, and S. H. Wei, Appl. Phys. Lett. 88, 042104 (2006).

[38] U. Celano, L. Goux, R. Degraeve, A. Fantini, O. Richard, H. Bender, M. Jurczak, and W. Vandervorst, Nano Lett. 15, 7970 (2015).

[39] C. Li, B. Gao, Y. Yao, X. Guan, X. Shen, Y. Wang, P. Huang, L. Liu, X. Liu, J. Li, C. Gu, J. Kang, and R. Yu, Adv. Mater. 29, 1602976 (2017).

[40] A. O’Hara, G. Bersuker, and A. A. Demkov, J. Appl. Phys. 115, 183703 (2014).

[41] A. C. M. Padilha and K. P. McKenna, http://dx.doi.org/ 10.15124/314ed070-65e2-466f-828e-f04d2c0de96d (2018) 